\title{
Mixing Enhancement of Opposed Quench Jets in a RQL Combustor
}

\author{
MAO Ronghai ${ }^{1^{\star}}$, SHEN Suhua ${ }^{1}$, GE Bing ${ }^{2}$, JI Yongbin $^{2}$ \\ ${ }^{1}$ AVIC Commercial Aircraft Engine Co., LTD., Shanghai 201108, China \\ *Corresponding author. Email: maoronghai@acae.com.cn \\ ${ }^{2}$ Key Laboratory for Power Machinery and Engineering of Ministry of Education, \\ Shanghai Jiaotong University, Shanghai 200240, China
}

Keywords: Combustor, RQL, Mixing, Jet, PLIF.

\begin{abstract}
Rich burn - Quick quench - Lean burn (RQL) combustion technology is one of the most promising low emission combustion technologies for aeroengines. During the whole process of RQL, rapid and effective mixing in the quenching zone is critical for its key role of bridging rich burn and lean burn processes. This paper carries out experiments and numerical simulation to investigate the mixing characteristics of the opposed quench jets on the annular RQL combustor simulator liners with the swirl flow. Acetone $\left(\mathrm{CH}_{3} \mathrm{COCH}_{3}\right)$ Planar Laser Induced Fluorescence (PLIF) is used to capture concentration distribution reflecting mixing of jets and main flow on the quench holes center section. The jet Reynolds number (1860-5580) and momentum flux ratio (0.045.76) are two key parameters investigated. Mixing non-uniformity is then analyzed based on the concentration results and non-swirl main flow conditions results were also obtained for comparison. CFD simulation model of the combustor test rig is created and Large-Eddy-Simulation (LES) model is adopted to demonstrate transient flow pattern details as well as to predict penetration trajectories. Results of mixing non-uniformity shows combination effect of the jet Reynolds number and momentum flux ratio, pointing out that optimal momentum flux ratio can be utilized to realize the most uniform mixing at certain jet Reynolds number. While for jet penetration performance, Reynolds number seems to make very little difference, which is mainly influenced by the momentum flux ratio. Besides, effect rule obtained from non-swirl mixing cannot be applied to jet mixing with swirling main flow.
\end{abstract}

\section{Introduction}

RQL combustion is a growing technology for gas turbine engines owing to its high combustion efficiency, lower emissions, enhanced flame stability, ignition and extinction performances, added fuel flexibility, and increased durability. NASA made efforts to design RQL combustors for the next generation aeroengines employing Low NOx emission combustion mode in $1990 \mathrm{~s}^{[1]}$. To realize the whole process of RQL, rapid and effective mixing in the quenching zone is extremely required for its key role of bridging rich burn and lean burn processes. For this reason, a large amount of studies focusing on the mechanism and factors of mixing jets into both reacting and non-reacting crossflow are carried out. Oechsle et al. ${ }^{[2]}$ reported that not much difference in temperature distribution was told between reacting and non-reacting cases by comparing mixing characteristics in a cylindrical duct. Shih ${ }^{[3]}$ numerically presented mixing process in the quenching zone and analyzed the effects of jet-mainstream momentum flux ratio on the mixing. Smith ${ }^{[4]}$ performed CFD calculation of jet mixing in reduced flow areas and found that although area-reduced quench zone would not enhance mixing, it would minimize residence time of the mixture so as to diminish NOx formation. Experimental work aiming at revealing the influence of amounts of quench holes on mixing was conducted by Leong ${ }^{[5]}$ in the cylindrical combustor, whose results showed depth of jets penetration was related to the number of holes. According to its correlation, optimization of holes number could be reached. Among investigated factors of mixing, momentum flux ratio is proved to be the most important aerodynamically, which is validated by the experiments of Zhu et al. ${ }^{[6]}$.

However, in real RQL combustors, the mainstream is swirl flow, which is quite different from the uniform mainstream cases discussed by most studies. Blomeyer ${ }^{[7]}$ investigated mixing characteristics experimentally in a rectangle RQL combustor and pointed out that the penetration depth of the jets in swirl cases is significantly decreased when compared non-swirl cases. Their 
study indicated that mainstream swirl is an important parameter and must be taken into account for the RQL quench design. Once the mainstream is swirling, mixing may not be only influenced by the momentum flux ratio under certain structures. Nevertheless, studies of jet mixing in swirl flow regarding to RQL combustor is still limited.

In terms of experimental methods, due to the remarkable advantage of PLIF (Planner Laser Induced Fluorescence) in displaying and measuring concentration field conveniently, it is widely used in studies of jet mixing ${ }^{[8-10]}$. It is a non-contact measurement technique utilizing the principle that specific substance in the flow will emit fluorescence when illuminated by the laser. As for fluorescence particles, acetone is an excellent choice for PLIF concentration distribution diagnose in gas cold flow. This owes to its superior properties: high fluorescence efficiency, low toxicity and cost, and more important is that acetone can be injected into the flow with extremely high concentration $^{[11]}$. Besides, numerical computation now is an important supplement part for tests, and LES (Large Eddy Simulation) becomes more preferred for gaining insight into flow details in gas turbine combustor ${ }^{[12,13]}$, as well as jet mixing process in the crossflow ${ }^{[14,15]}$, which is examined to show a good agreement with the experimental results.

This paper constructs a RQL model combustor (3/20 sectors of an annular chamber) and carries out visualization experiments of mixing characteristics with PLIF in non-reaction conditions, where acetone is adopted as fluorescence particles. At the same time, LES is implemented to get better understanding of the mixing process. The objective of this study is to reveal the effects of momentum flux ratio and jet Reynolds number on the mixing characteristics, mixing nonuniformity as well as penetration especially, in swirl flow. Difference between swirl and non-swirl is also discussed.

\section{Experimental Setup}

\subsection{Test Rig.}

Fig. 1 depicts the schematic view of the experimental system for the quench visualization measurement. The setup is mainly made up of four parts, mainstream system, quench air system, PLIF system and Test section. The mainstream at atmospheric pressure and temperature is supplied by a $7.5 \mathrm{KW}$ blower, whose flow rate is controlled by the frequency instrument adjusting rotation speed of the motor. In the quench air system, part of pressure air from the screw compressor is heated by the electric heater, and then transported into the pressure tank where liquid acetone as fluorescence particles is stored. In this way, high concentration level of acetone at certain temperature and pressure can be injected into the other part of pressure air as vapor phase with DANFOSS centrifugal atomizing nozzle easily. All the effort above is to ensure better atomization of acetone and mixing with the quench air. The test section is a sector combustor ( $3 / 20$ annulus) equipped with 3 axial swirlers and the two bended quench panels are installed on the outer and inner liner walls respectively. To obtain uniform quench injection, two plenums are set before the air is injected into the combustor.

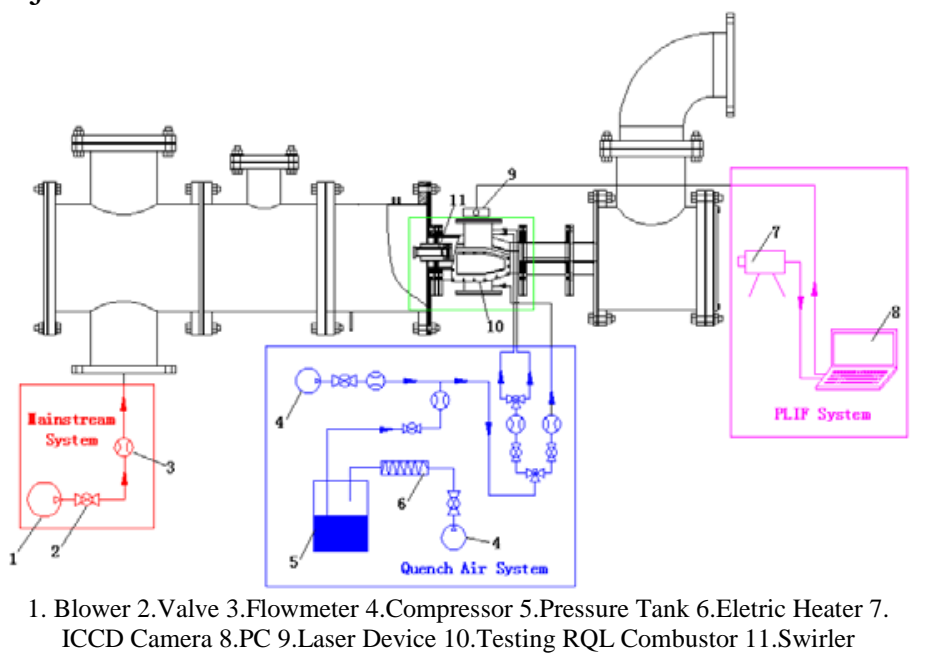

Fig.1 Schematic of the test rig system

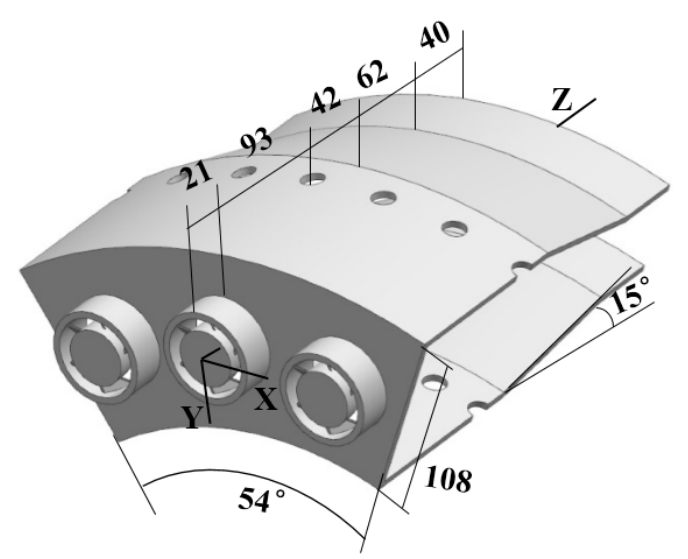

Fig.2 Schematic of combustor configuration 


\subsection{Combustor Configuration}

Fig.2 presents the RQL combustor simulator investigated in this study. The whole combustor is $248.5 \mathrm{~mm}$ in length, $108 \mathrm{~mm}$ in height and $54^{\circ}$ in span. The liner wall is composed by three kinds of panels in the streamwise direction. The first panel is $135 \mathrm{~mm}$ long, where quench units are located in $89 \mathrm{~mm}$ away from the outlet plane of the swirlers. Like the most annular combustor, the contraction structure formed by the second panel (62 $\mathrm{mm}$ long in streamwise direction) is adopted here, and the angle is $15^{\circ}$. The third panel is close the outlet of the combustor, and the length is $40 \mathrm{~mm}$. Round holes are served as quench units because of its convenience to realize even in real combustor, which are also the common investigation objects of most researches. The layout of 7 quench holes on the inner and outer liners respectively are opposed, whose diameters are all $14 \mathrm{~mm}$. Axial swirlers as flame stabilizer with 6 vanes of $45^{\circ}$ angle are utilized for the simulator, and the inner and outer diameters of the annular flow passage are $34 \mathrm{~mm}$ and $48 \mathrm{~mm}$, which means the hydraulic diameter of the combustor simulator inlet is $14 \mathrm{~mm}$.

\subsection{PLIF Measurement}

The hardware of PLIF measurements for mixing concentration field mainly consists of laser oscillator, synchronizer, acquisition device of fluorescence signal (ICCD camera) as well as other auxiliary system like optical lens. The pulse laser in 532nm band is triggered by the Quanta-Ray Lab-Series Nd:YAG laser device of Spectra-Physics, which works at the fixed frequency $(10 \mathrm{~Hz})$ with the highest $300 \mathrm{~mJ}$ output energy. Once trigged, the wavelength of the laser is changed by another tunable dye laser to meet the experiment demands. Rhodamine $6 \mathrm{G}$ is used as the dye to tune the original pump laser from $532 \mathrm{~nm}$ to the range of $559 \mathrm{~nm}-576 \mathrm{~nm}$ with the $0.0018 \mathrm{~nm}$ accuracy, and the max error is no more than $0.03 \mathrm{~nm}$. In PLIF tests, time interval between laser-triggered by the device and fluorescence signal captured by the ICCD camera as well as exposure time are controlled precisely by the SRSR DG535 digital pulse delay generator as the synchronizer. The pixel of the ICCD camera is $1024 * 1024$.

As shown in Fig.3, Laser sheet, shot into the simulator through the side quartz glass window, is vertical to the mainstream at the location of quench holes center plane ( $Z=114 \mathrm{~mm})$, and ICCD camera snapshots from the tail window. For the restriction of combustor structure and test rig, only $1 / 3$ zone of the quench mixing plane $(Z=114 \mathrm{~mm})$ can be pictured and analyzed, marked in Fig.4, which is actually reasonable for the periodicity of the flow pattern.

On account of the temperature and pressure in the combustor simulator nearly keep atmospheric $\left(20^{\circ} \mathrm{C}, 1 \mathrm{~atm}\right)$ during the experiment, which means that the gray value of every pixel in the PLIF image $I_{i}$ is proportional to concentration of fluorescence particle $C_{i}$. In this way, acetone concentration field represents the mixing state of the quench and mainstream. The gate width or exposure time of ICCD camera is set to 30ns to include a complete fluorescence for every instantaneous laser shot, and 100 single shot raw images are recorded for post-processing.

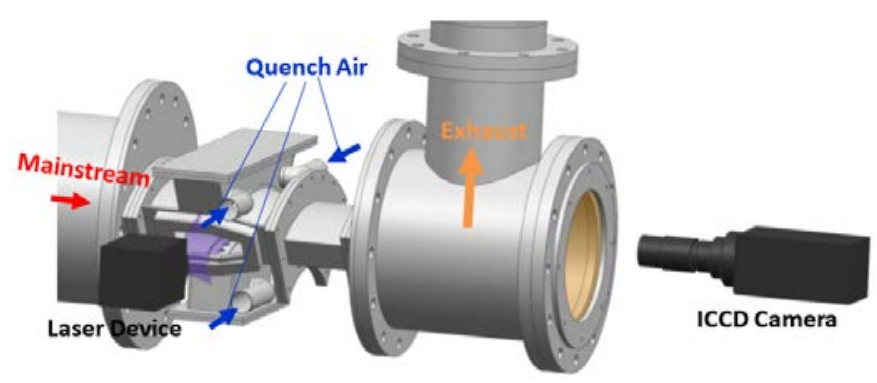

Fig.3 Schematic diagram of the PLIF photography

\subsection{Operating Conditions}

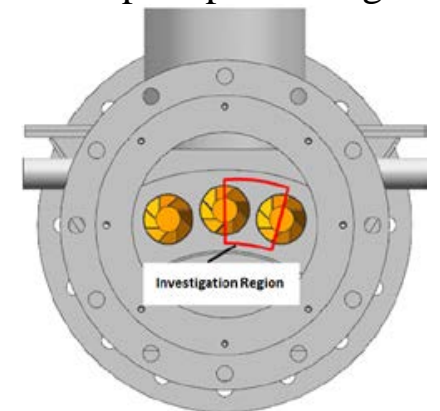

Fig.4 Schematic of shooting area

To reveal the swirl effect on the mixing characteristics, non-swirl cases under the same operating conditions are also carried out for comparisons. The experiments are performed using air, where the average jet Reynolds number $R e_{D}$ is 1860,3720 and 5580 , which is based on the quench jet diameter and jet velocity. Besides the momentum flux ratio $I$ based on the jet velocity $V_{Q}$ and mainstream inlet velocity $V_{M}$ varies ranging from 0.04 to 5.76 . The reasons why the momentum flux 
ratio seems small is that it is calculated based on $V_{M}$ for both swirl and no-swirl cases, which is easily controlled and compared. The test matrix is shown as following Table.1.

\begin{tabular}{ccccc}
\hline CASE & $\mathbf{v}_{Q}(\mathbf{m} / \mathbf{s})$ & $\mathbf{v}_{M}(\mathbf{m} / \mathbf{s})$ & $R e_{D}$ & $J$ \\
\hline $1-4$ & 2 & $2.5^{*}, 5,7.5,10$ & 1860 & $0.64,0.16$, \\
& & & & $0.07,0.04$ \\
$5-8$ & 4 & $2.5,5^{*}, 7.5,10$ & 3720 & $2.56,0.64$, \\
& & & & $0.28,0.16$ \\
$9-12$ & \multirow{2}{*}{6} & $2.5^{*}, 5^{*}, 7.5^{*}, 10^{*}$ & 5580 & $5.76,1.44$, \\
& & & & $0.64,0.36$ \\
\hline
\end{tabular}

Table 1 Test Matrix

Cases with * symbol (CASE 1,6,9,10,11,12) will be simulated numerically with Large-EddySimulation (LES) only for swirl mainstream to demonstrate more transient flow details, especially interactions between the mainstream and quench jets.

\section{Numerical Setup}

In this context, the commercial CFD code FLUENTR14.5 is used for LES simulation as supplementary to the experiments. In LES, large eddies are calculated directly by the filtered Navier-Stokes equations, the filter function, $G\left(x, x^{I}\right)$,

$$
G\left(x, x^{\prime}\right)= \begin{cases}1 / \Delta & \left|x-x^{\prime}\right|<\Delta / 2 \\ 0 & \left|x-x^{\prime}\right|>\Delta / 2\end{cases}
$$

where $\Delta$ is the volume of a computational cell. The filtered governing equations of continuity and momentum for incompressible fluid can be written as

$$
\begin{gathered}
\frac{\partial \bar{u}_{i}}{\partial x_{i}}=0 \\
\frac{\partial \bar{u}_{i}}{\partial t}+\frac{\partial}{\partial x_{j}}\left(\bar{u}_{i} \bar{u}_{j}\right)=-\frac{1}{\rho} \frac{\partial \bar{P}}{\partial x_{i}}+\frac{\partial}{\partial x_{j}}\left[v \frac{\partial \bar{u}_{i}}{\partial x_{i}}-\tau_{i j}\right]
\end{gathered}
$$

where the subscripts $i, j$ represent the spatial coordinates. $\tau_{i j}$ is the subgrid-scale (SGS) turbulent stress, and it can be expressed with SGS turbulent viscosity $\mu_{t}$.

$$
\tau_{i j}=-2 \mu_{t} \bar{S}_{i j}+\frac{2}{3} \delta_{i j} \tau_{k k}
$$

$\tau_{k k}$ is the isotropic part of the SGS stress, it will not be modeled but added to the pressure term. And $\bar{S}_{i j}$ is the rate of strain tensor.

$$
\bar{S}_{i j}=\frac{1}{2}\left(\frac{\partial \bar{u}_{i}}{\partial x_{j}}+\frac{\partial \bar{u}_{j}}{\partial x_{i}}\right)
$$

The SGS eddy viscosity $\mu_{t}$ is modeled by the Wall-Adapting Local Eddy-Viscosity (WALE) Model $^{[16]}$ in this study as

$$
\mu_{t}=\rho L_{s}{ }^{2} \frac{\left(S_{i j}{ }^{d} S_{i j}{ }^{d}\right)^{3 / 2}}{\left(\bar{S}_{i j} \bar{S}_{i j}\right)^{5 / 2}+\left(S_{i j}{ }^{d} S_{i j}{ }^{d}\right)^{5 / 4}}
$$

where $L_{s}$ and $S_{i j}^{d}$ are defined as

$$
\begin{gathered}
L_{s}=\min \left(\kappa d, C_{w} V^{1 / 3}\right) \\
S_{i j}{ }^{d}=\frac{1}{2}\left[\left(\frac{\partial \bar{u}_{i}}{\partial x_{j}}\right)^{2}+\left(\frac{\partial \bar{u}_{j}}{\partial x_{i}}\right)^{2}\right)-\frac{1}{3} \delta_{i j}\left(\frac{\partial \bar{u}_{k}}{\partial x_{k}}\right)^{2}
\end{gathered}
$$

where $C_{w}$ is the empirical constant determined by the experiments and set as 0.325 for it has been found to yield satisfactory results for a wide range flow. 
From the perspective of saving computation cost, only 1/20 sector of the whole annular combustor is modeled as the computational domain with the periodic boundaries. For its complexity, unconstructed grid is employed in the swirler part, while for the large scale chamber, where mainstream flow and quenching flow interact, constructed grid is the best choice, and they are merged on the interface. Moreover, Areas around quenching holes are the places where complex turbulent flow happens, and they are key parts affecting the quenching process, so mesh refinement is conducted here. After verifying the grid independence, 2 million is the final number of the grid size. The computation grid is showed in Fig.5, and boundary types are shown as well. Mainstream and quenching air inlet are all velocity inlets, two sidewalls are considered as periodic boundaries and outlet flow is assumed fully developed with atmospheric pressure. All other walls are no-slip and adiabatic walls. To demonstrate the mixing process, different temperature values are set for mainstream (293K) and quench jets (313K) inlet, and mixing behavior is represented using nondimensional temperature $\theta$ employed also by Morris ${ }^{[17]}$.

$$
\theta=\frac{T_{\text {main }}-T}{T_{\text {main }}-T_{j e t}}
$$

A second order central-differencing scheme is employed for spatial discretization. Temporal discretization is obtained using a second order implicit scheme and the time step is $20 \mu s$.

\section{Results and Discussion}

\subsection{Mixing non-uniformity}

As for PLIF measurement results, 200 instantaneous raw images are recorded and averaged to gain the time-averaged acetone concentration distribution. Normalized by the maximum concentration value, local relative concentration $C^{*}$ can be obtained at corresponding position, which is defined as:

$$
C_{i}^{*}=C_{i} / C_{\text {max }}=I_{i} / I_{\text {max }}
$$

Fig.6 shows the relative concentration distribution for CASE 6 without swirl flow and it is found that the $C^{*}$ around the quench holes is greater. This is because the quench jet keeps robust when just penetrates into the mainstream. With further penetration, the mixing between them becomes more intense, which accelerates the dissipation of the jet, hence the concentration reduces.

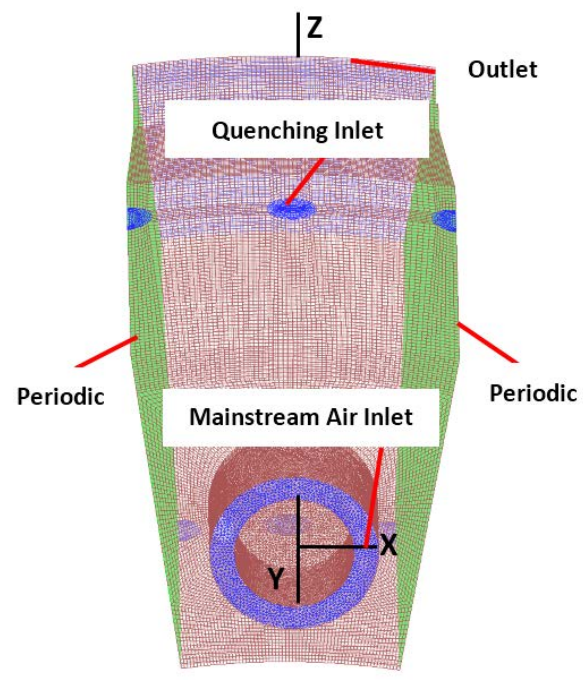

Fig.5 Schematic of the computational domain grid

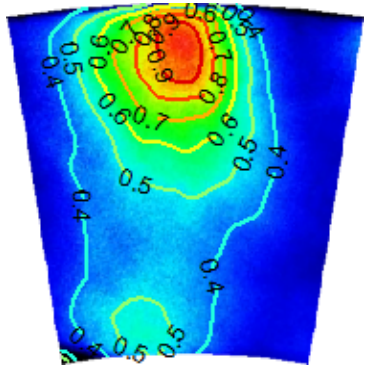

Fig.6 Schematic of time-averaged normalization acetone concentration distribution for CASE 6

And based on that, the mixing non-uniformity can be calculated as follows:

$$
\delta=\sqrt{\frac{1}{n} \sum_{1}^{n}\left(\frac{C_{i}^{*}-\overline{C^{*}}}{\overline{C^{*}}}\right)^{2}}
$$


where $\overline{C^{*}}$ and $n$ are the average relative concentration value and number of the pixels in the investigation region, respectively. $\delta$ varies in the $0-1$ range and the less $\delta$ presents, the more uniform mixing is realized.

\subsubsection{Effect of momentum flux ratio $J$}

As shown in Fig.7, for swirl flow cases, mixing non-uniformity $\delta$ is correlated with momentum flux ratio $J$ as well as jet Reynolds number $R e_{D}$. At a certain $R e_{D}$, taking $R e_{D}=1860$ for example, $\delta$ decreases along with the increasing logarithmic momentum flux ratio $l g J$, but with the further increase of $l g J, \delta$ reaches its minimum, and after that it begins to increase. It means that when jet velocity is kept constant, lower momentum flux ratio will obtain at a higher mainstream velocity, and in this case the mainstream swirling flow is dominant, jets are suppressed with limited penetration into the simulator. As a consequence, the mixing between two flows is weak, and mixing uniformity is worse. With the decreasing of the mainstream velocity, the momentum flux ratio gets increasing, resulting in drastic jets-mainstream interaction and increased turbulence, which is beneficial to the mixing uniformity. However, the further reduction of the mainstream velocity causes over penetration of the jets into the main flow. At the moment, the momentum of the quench flows overweighs that of main flow a lot, jets become absolutely dominating, which implies that the dissipation of the jets in the mainstream are weakened. So the mixing seems less uniform. The trend is the same in the other two $R e_{D}$ cases.

\subsubsection{Effect of jet Reynolds number $R e_{D}$}

Concerning the jet Reynolds number $R e_{D}$ effect on the mixing non-uniformity, it is more complicated. In the experimental investigation parameter range, there are four different regions associated with $R e_{D}$, split by the 3 black lines shown in Fig.11. In the first region, when $I$ is relatively small, $\delta$ increases with the increased $R e_{D}$, which means lower quench jets velocity brings about more uniform mixing field. Moving to the second region, momentum flux ratio gets increased but still less than 1, and $\delta$ for $R e_{D}=3720$ is lower than that for $R e_{D}=1860$ and 5580. Taking experiments results of $I=0.64$ for example, $\delta$ for $R e_{D}=1860$ and 5580 are $21 \%$ and $57 \%$ higher than that for $R e_{D}=3720$. And it still keeps most uniform mixing in the third region at $R e_{D}=3720$, but the $R e_{D}=1860$ case is least non-uniform. In the last region with larger momentum flux ratio, mixing nonuniformity is increased with decreasing $R e_{D}$.

Actually, through fitting with experimental data at certain $R e_{D}$, it is found that correlation between and $\delta$ and $l g J$ is quadratic polynomial, pointing out the existence of optimal $I$ making the most uniform mixing for every $R e_{D}$. And the optimal momentum flux ratio rises as $R e_{D}$ increases.

\subsubsection{Effect of swirl}

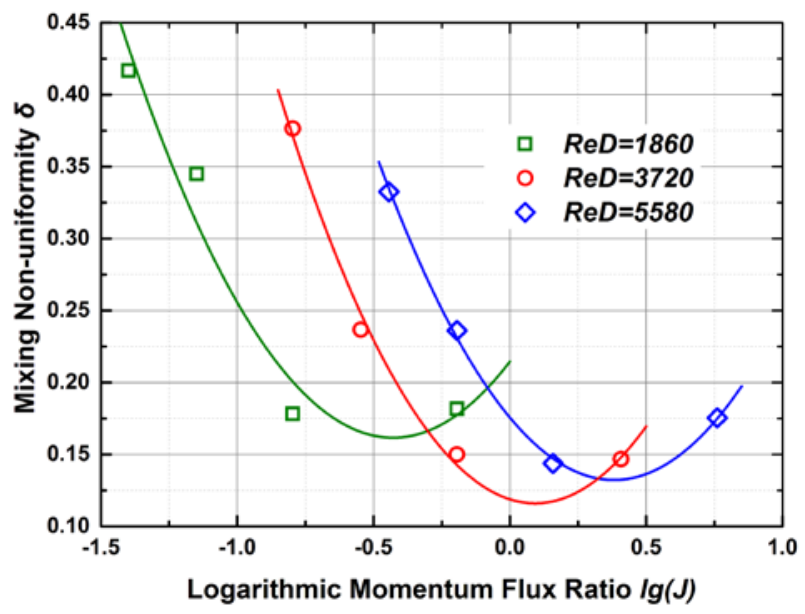

Fig.7 Mixing non-uniformity for all swirl flow cases

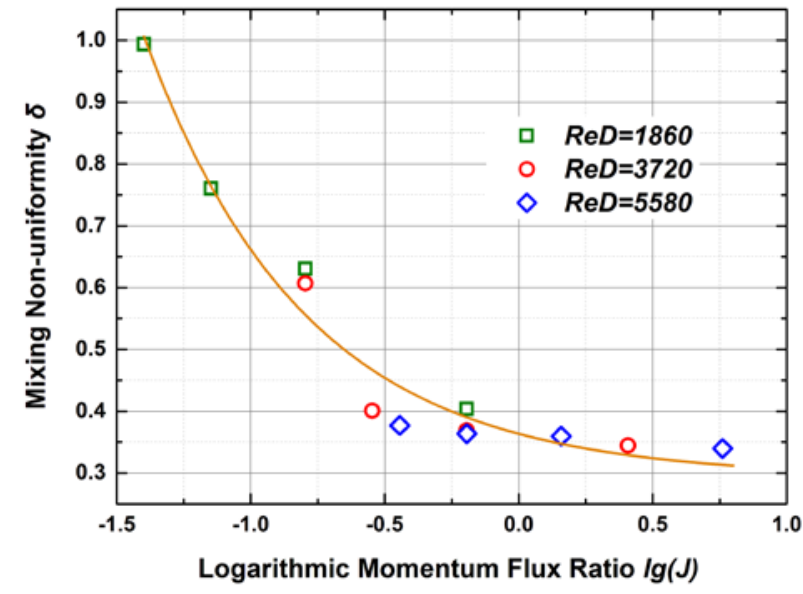

Fig.8 Mixing non-uniformity for all non-swirl flow cases

It is another totally different situation for non-swirl mainstream cases, which has been thoroughly investigated as discussed in literature review. Fig.8 shows the relation with respect to the mixing non-uniformity and momentum flux ratio as well as jet Reynolds number. It implies that the mixing uniformity is only decided by the momentum flux ratio, having nothing to do with $R e_{D}$. The value of $I$ is the same (0.16) for both Case $2\left(R e_{D}=1860\right)$ and Case $8\left(R e_{D}=3720\right)$, and a huge 
difference of $110 \%$ for $\delta$ in the swirl condition, while only $4 \%$ in the non-swirl condition. Moreover, even with the same $I$ and $R e_{D}, \delta$ is larger when the main flow is non-swirling, which indicates swirl mainstream flow characterized with tangential momentum will promote a more uniform mixing with quench jets. This is what RQL requires for quick mixing.

Fig.9 shows PLIF instantaneous images for CASE 5, what can be clearly seen from the contrast is that the border for acetone concentration standing for quench jets in non-swirl crossflow is distinct, while it is quite blurred in swirl crossflow. It reveals that interaction of injected flows with swirl mainstream is much more intense than that with non-swirl mainstream, this explains why the mixing in swirl cases is more uniform.

\subsection{Flow characteristics analysis}

Concluded from the above analysis, complicated flow pattern in the swirl-stabilized combustor simulator influences the mixing behavior mainly, which needs detailed analyses to understand mixing thoroughly. Simulation shows its incomparable advantage in this respect, especially LES can give transient flow characteristics to observe development of the flow.

\subsubsection{Flow structures}

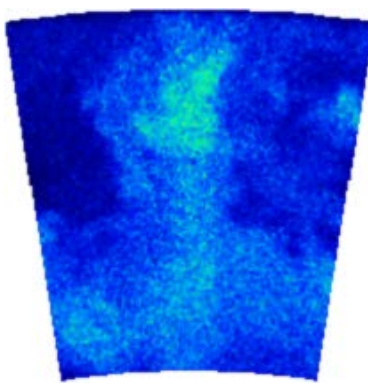

(a) non-swirl

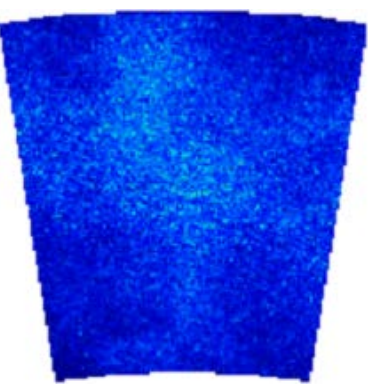

(b) swirl

Fig.9 PLIF instantaneous images for CASE5

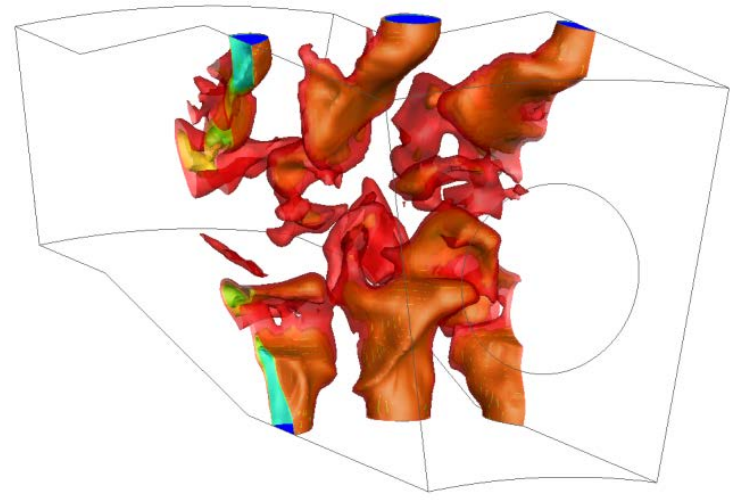

Fig.10 Iso-nondimensional temperature magnitude distribution

Fig.10 presents the iso-nondimensional temperature $\Theta$ distribution showing that jets are deflected and twisted after entering into the combustor for the reason of the tangential momentum component of the swirling main flow. It is so tense that vortex is induced in the interaction region, and developed towards to the surrounding as well as downstream, which promotes the uniform mixing. Besides, quench holes on the inner liner are more compact than that on the outer liner, so the interference is also stronger. What's more, quench jets into the simulator from the inner liner are divergent, while it is convergent to one point for the outer liner jets. This is why the mixing of opposed quench jets in annular combustor seems not symmetric in the swirling conditions, which is quite different from previous traditional studies on rectangular and circular combustors.

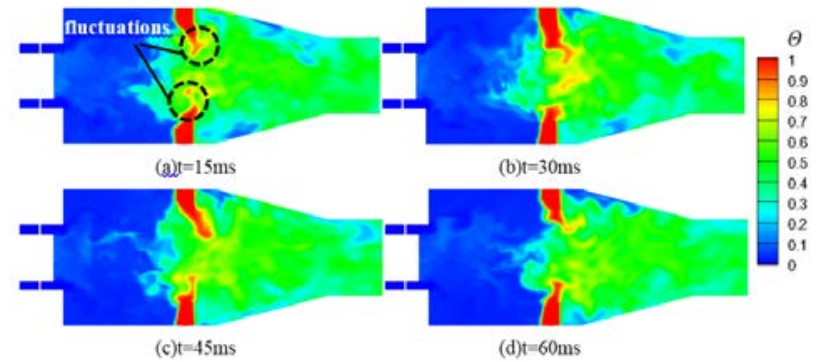

Fig.11 Time sequence images of nondimensional temperature(CASE 6)

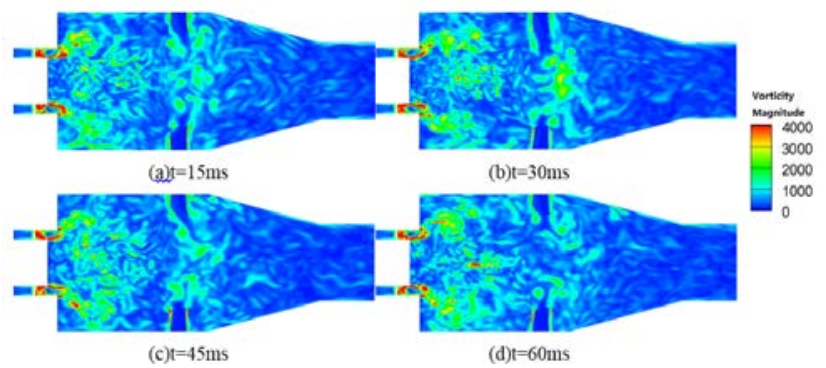

Fig.12 Time sequence images of vorticity magnitude(CASE 6)

Time sequence images $(\mathrm{t}=15,30,45,60 \mathrm{~ms})$ of non-dimensional temperature field on $\mathrm{X}=0$ plane are illustrated in Fig.11. Quench jets get deeper penetration into the mainstream with more and more intense intervention, producing fluctuations at the end of the jets. As time goes on, eddies induced by shear effect fluctuations shed and dissipate gradually along with the flow development towards to the downstream direction. More apparent proof of vorticity distribution depicted in Fig.12 correlate the above discussed process. 


\subsubsection{Jet trajectory}

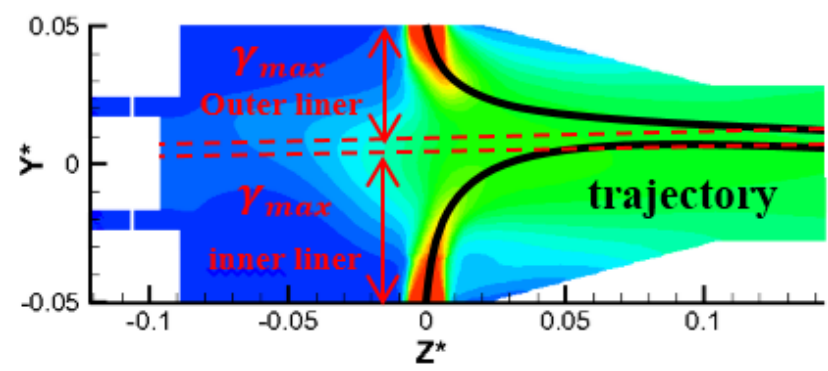

Fig.13 Schematic of definition of jets trajectory and maximum penetration depth

As defined by Norster ${ }^{[18]}$, jet trajectory can be captured by taking temperature traverses at various distances downstream of jets exit in the vertical section and viewing the lowest temperature points as the jet centers, which are lined as trajectory. And the maximum penetration depth $\gamma_{\max }$ is the depth at which the jet centerline reaches asymptotic to the main flow, illustrated schematically in Fig.13.

Bases on the definition, jet trajectories for all cases are calculated by time averaging nondimensional temperature distribution, which are shown in Fig.14 as well as Fig.15. As can be seen from the Fig.14, at certain momentum flux ratio ( $\mathrm{J}=0.64)$, jet trajectories along the non-dimensional $\mathrm{Z}$ direction appear to have little difference with jet Reynolds number $R e_{D}$ varying from 1860 to 5580 . While Fig.15 shows that jets trajectories comparison for different $\mathrm{J}$ with the constant jet Reynolds number $R e_{D}=5580$. The trend is that jet penetration depth increases with the increasing $I$, while the increase rate is decreasing and it reaches $\gamma_{\max }$ faster for higher $I$. Experimental results discussion above has indicated that there is an optimal momentum flux ratio to obtain most uniform mixing for selected $R e_{D}$. Likewise, from the perspective of jets penetration, momentum flux ratio also needs to optimize carefully for the reason that it is very important to ensure rational jets penetration for realizing quench process successfully. Too little $J$ is not enough for jets entering into mainstream flow for mixing but rather rapid dissipation, on the contrary, too large $I$ will cause oppose quench jets to impinge with each other, developing into jet collision with limited jets-main flow interaction. To sum up, trajectory and maximum penetration for quench jets in swirling flow are only up to momentum flux ratio and unaffected by $R e_{D}$ variation. Furthermore, trajectories for quench jets from outer liner and inner liner are not symmetrical, and inner liner seems beneficial for deeper jets penetrating. The velocity of mainstream flow near the inner liner is lower, resulting in higher local momentum flux ratio and deeper penetration consequently.

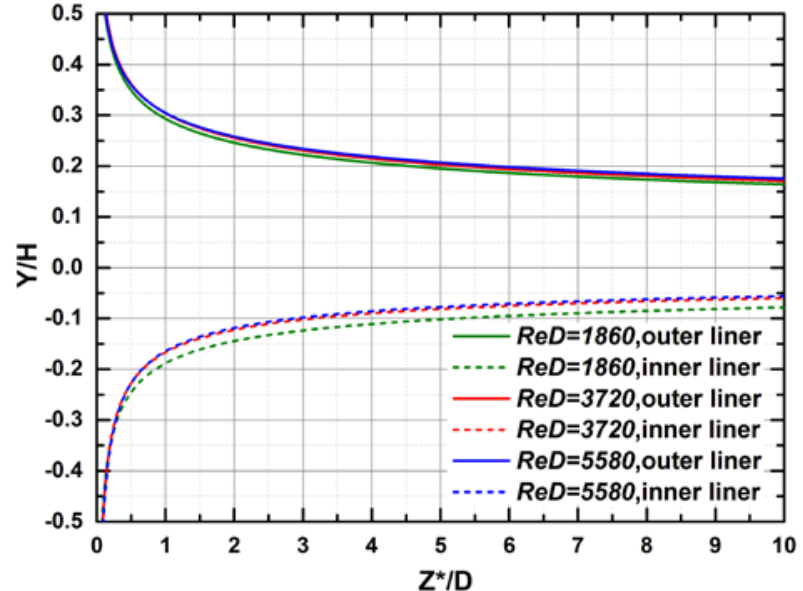

Fig.14 $\operatorname{Re}_{D}$ effect on Jet trajectories $(I=0.64)$

\section{Conclusions}

A RQL annular combustor simulator with one row of round quench holes as well as three axial swirlers was constructed. PLIF laser diagnostic experiments on mixing non-uniformity and LES on detailed mixing process of jets with swirling flow including penetration trajectories were performed at different jets Reynolds number $R e_{D}$ ranging from 1860 to 5580 and momentum flux ratio $l$ ranging from 0.04 to 5.76. Experimental results show that at certain $R e_{D}$ mixing non-uniformity $\delta$ decreases 
with $I$ at first, while after reaching a certain amount of $I, \delta$ begins to increase, which suggests an optimal $I$ making the most uniform mixing for every $R e_{D}$. While it is totally different for non-swirl conditions where $\delta$ is only correlated with $I$, having nothing to do with $R e_{D}$. Besides, $\delta$ is larger for non-swirl mixing generally, indicating that swirl mainstream flow characterized with tangential momentum will promote a more uniform mixing with quench jets, and rule obtained from non-swirl mixing is not applicable to jets mixing with swirling main flow.

Transient mixing process of opposed quench jets with swirl flow is predicted successfully by LES. It clearly shows the interaction between the jets and main flow and jets deflection especially. Unlike mixing non-uniformity, jet trajectories along the non-dimensional Z direction appear to have little difference with $R e_{D}$, whereas it is only decided by $I$. Jet penetration depth increases with the increasing $I$. In addition, trajectories for quench jets from outer liner and inner liner are not symmetrical as a result of different local momentum flux ratio.

In conclusion, mixing of quench jets with swirl flow is quite important for realizing Rich burnQuick quench-Lean burn functions. Mixing characteristics mainly including non-uniformity and penetration are effected by the combination of $R e_{D}$ and $I$. Even under certain combustor structure, optimization mixing can be reached by adjusting flow conditions.

Acknowledgements. Financial support from Science and Technology Commission of Shanghai Municipality, Pujiang Program 13PJ1432700 and 13DZ2281200, is greatly acknowledged.

\section{References}

[1]. Dennis R, The gas turbine handbook. In: Samuelsen GS(ed) Conventional Type Combustion. 2006, http://seca.doe.gov/technologies/coalpower/turbines/refshelf/handbook/3.2.1.1.pdf

[2]. Oechsle VL, Mongia HC, Holdeman JD, Comparison of mixing calculations for reacting and non-reacting flows in a cylindrical duct. In: 32nd Aerospace Sciences Meeting and Exhibit, 1994, AIAA-94-0865

[3]. Shih TIP, Nguyen HL, Howe GW, Li Z (1991) Simulation of mixing in the quick quench region of a rich burn-quick quench mix-lean burn combustor. In: 29th Aerospace Sciences Meeting, AIAA-91-0410

[4]. Smith CE, Talpallikar MV, Holdeman JD, A CFD study of jet mixing in reduced flow areas for lower combustor emissions. In: 27th AIAA/SAE/ASME/ASEE Joint Propulsion Conference, 1991, AIAA-912460

[5]. Leong MY, Samuelsen GS, Holdeman JD, Optimization of jet mixing into a rich, reacting crossflow. J Propul Power 16, 2000:729-735

[6]. Zhu G, Lai MC, Lee T, Penetration and mixing of radial jets in neck-down cylindrical crossflow. J Propul Power 11, 1995:252-260

[7]. Blomeyer M, Krautkremer B, Hennecke DK, Mixing zone optimization of a Rich-Burn /Quick-Mix/ LeanBurn combustor. J Propul Power 15, 1999:288-295

[8]. Law AWK, Wang HW, Measurement of mixing processes with combined digital particle image velocimetry and planer laser induced fluorescence. Exp Therm Fluid Sci 22, 2000:213-229

[9]. Shao DD, Law AWK (2010) Mixing and boundary interactions of $30^{\circ}$ and $45^{\circ}$ inclined dense jets. Environ Fluid Mech 10:521-553

[10]. Sherwin DJ, Koch JD (2014) In situ PLIF and particle image velocimetry measurements of the primary entrainment fuel jet in a naturally aspirated water heater. J Fluid Eng 136:021104-1-21104-6

[11].Thurber MC, Grisch F, Kirby BJ, Votsmeier M, Measurements and modeling of acetone laser-induced fluorescence with implications for temperature-imaging diagnostics. Appl Optics 37, 1998:4963-4978

[12]. Grinstein FF, Fureby C, LES studies of the flow in a swirl gas combustor. P Combust Inst 30, 2005:17911798

[13].Patil S, Tafti D, Large eddy simulation of flow and convective heat transfer in a gas turbine can combustor with synthetic inlet turbulence. In: Proceedings of ASME Turbo Expo 2011, GT2011-46561

[14]. Recker E, Bosschaerts W, Large eddy simulation of mixing in a round jet in cross-flow. In: 39th AIAA Fluid Dynamics Conference, 2009: AIAA 2009-3561

[15]. Nagao T, Matsuno S, Hayashi AK, Fluid mixing of opposed jet flows in the rectangular duct. In: 51st AIAA Aerospace Sciences Meeting including New Horizons Forum and Aerospace Exposition, 2013: AIAA-20130872

[16]. Nicoud F, Ducros F, Subgrid-scale stress modelling based on the square of the velocity gradient tensor flow. Flow Turbul Combust 62, 1999: 183-200

[17]. Morris RM, Snyman JA, Meyer JP, Jets in crossflow mixing analysis using computational fluid dynamics and mathematical optimization. J Propul Power 23,2007: 618-628 
[18]. Arthur H. Lefebvre, Dilip R. Ballal, Gas Turbine Combustion: Alternative Fuels and Emissions, $3^{\text {rd }}$, 2010:125. 